\title{
Evaluation of Environmental Monitoring Strategies
}

In environmental monitoring, it is important that the monitoring system should emit early warnings when undesired events occur. These events may be sudden or of a more subtle nature. In the design of such monitoring systems, a proper balance between cost and risk must be achieved. There are 2 classic types of risk connected with early warning systems, namely the risk of not detecting significant changes and the risk of false alarms. The purpose of this paper is to describe a method for comparing the performance of different monitoring systems, considering the classic types of risk and cost. The method is applied to the monitoring of the lichen cover as a test case. The expected utility has been used as a measure of performance. When estimating the probabilities of the events, spatial microsimulation and Monte-Carlo simulation techniques have been used. The monitoring programs studied are based on satellite images, aerial photos, field samples, and land-cover maps. The major conclusions of this study are that standardized quality measures are extremely useful for evaluating the usability of environmental monitoring methods. In addition, when estimating gains and costs, spatial microsimulation techniques are useful. To improve the method, however, macroconstraints should also be used for aligning the simulation model.

\section{INTRODUCTION}

During the last 15-20 years, vegetation changes and degradation have been reported in the mountainous areas of Sweden. The Swedish Environmental Protection Agency (Swedish EPA) intends to establish a system for monitoring environmental quality where "a magnificent mountain landscape" is one of the objectives (1). To achieve this objective there is a need to determine a strategy for monitoring changes in the vegetation cover (2).

Irrespective of the design of the monitoring system, it is important that the system should emit early warnings when undesired events occur. The detection of changes can form part of a decision-support system concerning actions to reduce land degradation. In general, demands on the mountain areas have increased during the last few decades. Tourism continues to increase, off-road vehicles have become common, and reindeer management methods have, since the mid-1970s, become more efficient and motorized (3). The combined effect of climate change and continued high human impact could have a severe negative effect on the long-term sustainable development of mountain areas in Sweden.

The purpose of this paper is to describe a method for comparing the performance of different monitoring systems. Special emphasis is placed on evaluating the risks associated with the different designs and on their related costs and benefits. Standards related to quality descriptions of source data form the basis of the developed method. The method is applied to the monitoring of the lichen cover as a test case.
Cornélis and Brunet (4) discuss a generic model for decision making and the uncertainties that occur along the entire decisional process. In any decisional process, decision making is based on a decision analysis phase, which in turn relies on a documentation/information phase. The uncertainties in the decision-making phase are mainly due to its procedures and to the nature of human beings, whereas the uncertainties in the analysis phase originate from the chosen method and the way in which reality is simplified. Often the decision-makers face widely varying types of information, with each type being associated with its own uncertainties. According to Cornélis and Brunet (4) public decisions are all spatially referenced, if not spatially induced, since they apply to a specific territory.

When designing a monitoring program, the most important factors to consider may be the certainty of its predictions and the cost of the program. Since a monitoring program can be designed in several different ways, it is important to achieve a proper balance between these aspects. Different designs may include source data from many disciplines, which may have their own definitions and approaches for dealing with uncertainty. Consequently, the designer is faced with estimating the various contributions of uncertainty from each of these sources, as well as with integrating their disparate terminology (5).

The importance of spatial data quality has received much attention during the past 15 years. However, most past research seems to focus on the presence of errors in source data and the need for standards regarding the data quality components. In addition to these standardized quality components concerning the source data, de facto standards concerning, for instance, air-photo interpretation and satellite image interpretation have appeared. For example, the confusion matrix used to evaluate remote sensing classification is a well-accepted description of uncertainty in source data. The development of this confusion matrix is termed "accuracy assessment", because classification errors expressed therein are with respect to some reference data (6). The confusion matrix is indicative of errors of omission and commission found in the map and is a starting point for various descriptive and analytical statistical techniques. In addition to its use with remotely sensed data, the confusion matrix or contingency table approach is appropriate to other spatial data applications (5). It is, however, important to keep in mind that the confusion matrix is based on training data. As a consequence, such information only indicates how well the statistics from these areas can be used to categorize the same areas; i.e. such information gives little indication of how the classifier performs elsewhere in a scene and, therefore, one should expect training area accuracies to be overly optimistic (7).

Another accepted description of the distribution of a category's spectral response pattern is the mean vector in combination with the covariance matrix. If these parameters are given, it is possible to compute the statistical probability 
of a given pixel value being a member of a particular landcover class (7).

A very active area of research and development in the geographic data community is the documentation of data in the concept of metadata, i.e. data about data. Metadata may contain handling instructions for data, such as details of its format, information about its quality, and information needed by the user to determine its fitness for use (8).

The ISO approach to evaluating spatial data quality can be described as follows (9). By using a database specification, a universe of discourse (perfect dataset), $P^{*}$, is defined. The quality of the actual data being used, $\hat{P}$, may then be expressed as

$\bar{Q}_{P}=\operatorname{Dist}\left(\hat{P}-P^{*}\right)$

where $\bar{Q}_{P}$ is an n-dimensional vector, expressed by standardized measures such as accuracy, completeness, etc.

Dist is a distance function in an n-dimensional space.

A user wishing to evaluate the suitability of $\hat{P}$ should according to the ISO model specify his or her universe of discourse $\left(U^{*}\right)$. Then the dataset is suitable if

$\bar{Q}_{U}=\operatorname{Dist}\left(\hat{P}-U^{*}\right)<\bar{\varepsilon}$

So far there is limited experience of applying the ISO model. The main problems seem to be:

- How is $\bar{Q}_{P}$ estimated? This problem increases as the semantic differences between $P^{*}$ and $U^{*}$ increase.

- What is a suitable threshold vector, $\bar{\varepsilon}$ ? How are the errors and uncertainties propagated to the final result?

To date, uncertainty methods in the decision phase have been pointed out by some studies, but examples of applications of such methods in this area are still quite few. A novel procedure to analyze the uncertainty associated with the output of GIS-based models is presented by Crosetto and Tarantola (10); i.e. a synergetic use of uncertainty analysis and sensitivity analysis is proposed. Crosetto and Tarantola briefly discuss how the procedure can provide a model building tool for choosing, from a group of alternative models, the best model in terms of cost-benefit analysis. In their case study, they illustrate how uncertainty analysis and sensitivity analysis can support the implementation of a GIS-based hydrologic model whose main goal is to support a system for near real-time flood forecasting. DeBruin et al. (11) propose a decision-analytical approach to compare the value of alternative datasets. Their case study concerns the expected monetary loss due to uncertainty concerning the volume of sand required for building a container port. Aerts (12) studies the influence of uncertainty on the potential locations of a ski run and its associated development costs.

Frank and Grünbacher (13) discuss the relevance of datasets. They state that the relevance of data can be decided only with respect to a decision. One test of relevance is to study whether the decision has the same outcome if the dataset is improved or degraded. They continue by suggesting a formal framework for the revision of datasets. Krek (14) presents an agent-based computational model for quantifying the functional value of spatial information and applies it to car navigation as a case of spatial decision making.

An alternative approach to the ISO model is to study the effect of uncertain and incomplete information in the decision domain. Assuming, for simplicity reasons, that we are making rational decisions based solely on facts (information), then the decision-making process can be expressed as a function:

$d=f(U)$

where $d \in D, D$ constitute the set of all possible decisions, and $U$ is the information available.

An optimal decision may then be written as

$d^{*}=f\left(U^{*}\right)$

The suitability of the dataset $\hat{P}$ may then be expressed as

$\bar{S}_{\hat{p}}=\operatorname{Dist}_{2}\left(\hat{d}-d^{*}\right)=\operatorname{Dist}_{2}\left(f(\hat{P})-f\left(U^{*}\right)\right)$

where $\bar{S}_{\hat{P}}$ is an n-dimensional vector describing the suitability. Dist $_{2}$ is a distance function in an n-dimensional space, expressing the difference between two decisions.

The problems still to be solved with this approach are the following:

- Given a set of optimal facts $\left(U^{*}\right)$, what is the optimal decision?

- Given a set of information, $\hat{P}$, what is the actual decision being made?

- How should the difference between two decisions be expressed?

This paper deals with problems related to quality assessment in the decision domain. The idea is that existing standards related to quality descriptors can be used for such assessment. The purpose is to study whether quality specifications can be used for the evaluation of different alternatives within a decision-making process concerning its risks and associated costs.

The case study described in this paper consists of 3 steps. In the first step, the perfect datasets $U^{*}$ are produced, using a spatial microsimulation engine based on cellular automata. In the second step, the datasets $\hat{P}$ are generated using Monte Carlo simulation. Finally, in the third step, the suitability of the monitoring methods is evaluated using expected utility measures.

\section{MATERIALS AND METHODS}

\section{Systems for Monitoring the Lichen Cover}

Monitoring all the changes associated with vegetation is impossible. In order to accomplish the aim of monitoring changes in vegetation, it is necessary to establish one or more indicators of change in the vegetation cover. A good indicator will simplify large amounts of complex information into a concise, easily understood format. To be effective, an indicator must be relevant, easy to understand and reliable. In the present case study, lichens were used as an indicator of ecosystem disturbance.

Of the different heath communities above the treeline, extremely dry and dry mountainous heaths and lichendominated dry heaths are vulnerable to mechanical damage, such as that caused by trampling by animals or humans, or by tyre friction made by off-road vehicles (15). If such mechanical damage becomes continuous, the consequence is vegetation degradation and soil erosion. Soil erosion can be 

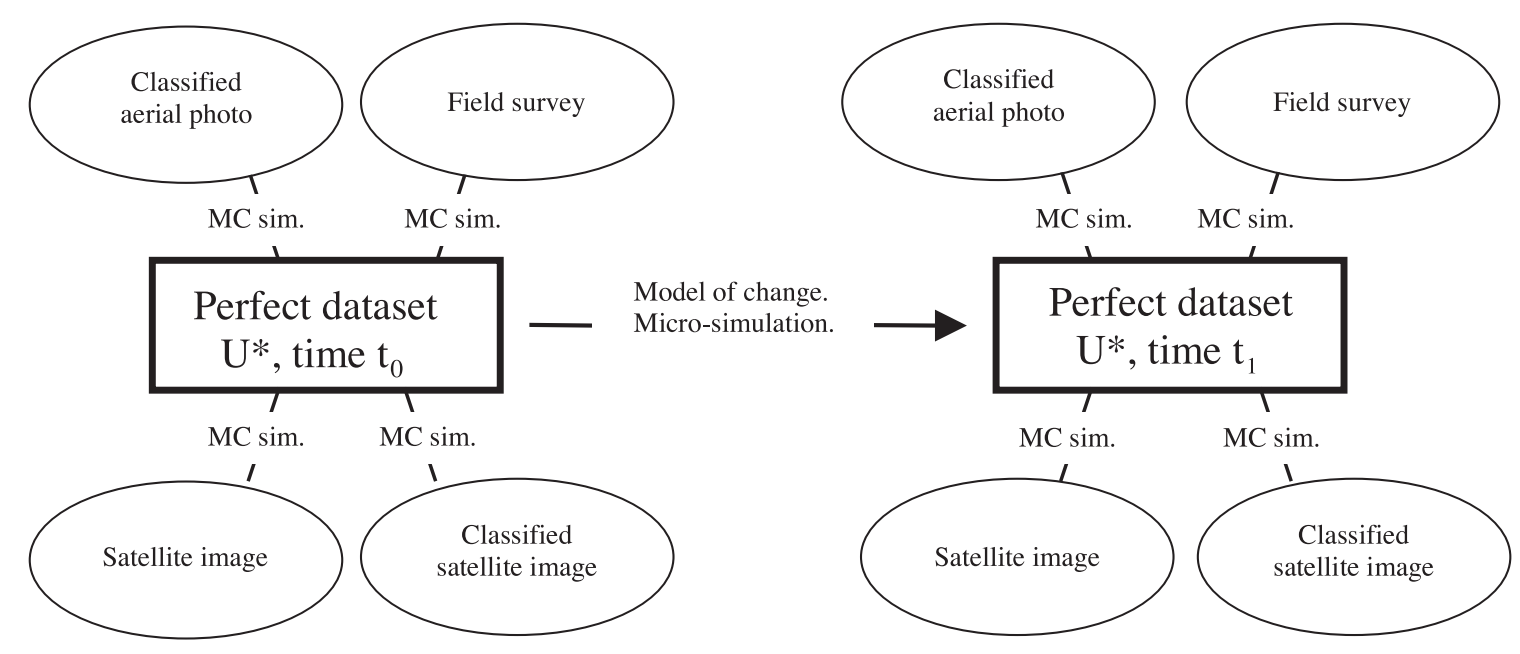

Figure 1. A schematic overview of the laboratory environment.

seen in the increased number of patches of exposed mineral soils in drier vegetation (16). Extremely dry heaths are found in locations that are often swept free from snow in the winter. Dry heaths occur where the snow cover is better, and are dominated by crowberry (Empetrum hermaphroditum) and, where calcareous soils are rare, dominated by heather (Calluna vulgaris). In areas with a continental type of climate, lichens often dominate the dry heath. In dry conditions, lichens are brittle and vulnerable to trampling $(17,18)$.

Using Landsat TM data, Tömmervik and Lauknes (19) showed using Landsat TM data, that one of the main vegetation changes observed in the land cover in northern Norway was the decrease of lichen in lichen-dominated heaths. Pale lichens may be used as a remote-sensing indicator of change, since due to their spectral characteristics they differ clearly from green vegetation, by reflecting highly in the visual and infra-red wavelength bands. For monitoring Swedish mountainous vegetation in areas with a continental climate and dominated by lichen-rich heaths, the chosen indicator (lichen cover) can be used for detection of disturbances in the ecosystem; and changes in the lichen cover are possible to detect on a regional scale in Landsat TM data using an NDVI differencing technique (2). Using the same indicator Allard (16) showed that small-scale changes in the lichen cover are possible to detect through interpreting Colour Infra-Red (CIR) aerial photographs with a scale of 1:60 000 from 2 periods representing a time-span of about 15 years.

Four different methods for estimating the lichen cover were used in this study, namely

- interpretation of aerial infra-red images;

- classification of TM data;

- spectral changes in TM data;

- field measurements.

Using the first method, color infra-red aerial photographs with a scale of 1:60 000 were interpreted for 2 different dates. Within these interpreted photographs the percentage of the lichen cover was estimated. A difference image was then calculated to illustrate the lichen change. Since Allard et al. (20) show that the lichen cover can be estimated to the nearest $10 \%$, their findings were implemented.

The second method compares 2 classifications of Landsat TM data. According to Singh (21) this post-classification method can produce a large number of erroneous change indications, since an error on either date gives a false indication of change. However, the method holds promise, because data from 2 dates are separately classified, thereby minimizing the problem of normalizing for atmospheric and sensor differences between 2 dates.

In the third method the change detection using satellite images is based on calculations of the normalized difference vegetation index (NDVI). The created difference image shows the change as NDVI differences. Linear regression is then used to calculate the relationship between the NDVI differences and the lichen decrease interpreted using the CIR aerial photograph. The reason for using this NDVI method is that studies by Nordberg and Allard (2) show that the NDVI differencing technique and thresholding can be used for detecting changes in the lichen cover with an overall accuracy of $77 \%$. These findings were used as a base when designing an NDVI-based monitoring system for the present case study.

The method based on field measurements was chosen, since fieldwork is in most cases carried out to acquire detailed information on the ground cover, as well as to obtain detailed and reliable reference data when evaluating a developed method. Several areas that represented homogenous sites of different character were selected in an image to be visited and checked.

These monitoring methods were selected because they had been prepared and were available within the researchgroup. That they represent the best change detection techniques has not been verified. The methods were implemented in a laboratory environment for further studies. The analyses made were based on artificial perfect datasets $\left(U^{*}\right)$. However, these artificial data were based on existing datasets for an area in a Swedish mountain range. In Figure 1, a schematic overview of the laboratory environment is shown.

\section{Construction of Perfect Datasets $\left(U^{*}\right)$}

To study environmental change, data from at least 2 different times should be used. The perfect datasets required for this purpose are

$$
U^{*}=U_{0}^{*} \cup U_{1}^{*}
$$

First of all an artificial perfect dataset for time $t_{0}$ was constructed $\left(\mathrm{U}_{0}{ }^{*}\right)$ on the basis of the classification of existing 
real datasets. From this perfect dataset, 2 different raster images were generated. One image had 13 different landcover classes, one of which represented lichen heath, while the other image had the lichen heath class divided into 9 subclasses, depending on the amount of lichen-domination.

In order to produce a perfect dataset for time $\mathrm{t}_{1}\left(U_{1}{ }^{*}\right)$, the changes in the lichen cover have to be reflected. Changes in the lichen cover depend on damage as well as on growth. Since the growth of lichen is assumed to take 30 years (22), time $t_{1}$ was set to $t_{0}+30$ years. To produce $U_{1}^{*}$, a spatial microsimulation technique was used.

Micro-simulation originates from the social sciences and has been used for modelling different aspects of the social world, like demographic, social, or economic characteristics of human behavior. The prefix "micro" simply indicates that the simulation model is formulated at a disaggregate level or microlevel of individual decision making, i.e. for units such as individual persons, households, or vehicles $(23,24)$. While macromodelling usually provides good estimates of mean values, microsimulation is used when the entire distribution functions are of interest or when rare events are to be modelled (24).

In this study, the main causes of damage to the lichen cover were assumed to originate from trampling by humans and reindeers. Depending on the precipitation, this trampling will cause various degrees of damage. Growth is assumed to be a linear function over the time period; i.e. the model of changes in the lichen cover was very much simplified in this study. Hence, 3 different functions for changes were created and used.

For the 3 different models of change, variables describing the trampling by humans and reindeers and the precipitation were randomly changed. Each model of change was then reprocessed 10 times, i.e. 30 different, equally likely, alternatives for $U_{1}^{*}$ were generated.

\section{Construction of Artificial Datasets $(\hat{P})$}

The method suggested for comparing different monitoring strategies uses quality specifications to create aerial photo interpretations and classified satellite images. Since such specifications were missing for some monitoring strategies, adequate quality measures were estimated by other means, for example interviews.

Classified aerial photo interpretations were simulated from the perfect datasets $\left(U_{0}^{*}\right.$ and $\left.U_{1}^{*}\right)$, with the lichen class divided into 9 subclasses. The confusion matrix created for photo interpretation was used for this purpose. It was assumed that misclassification only occurs for complete areas, not just one pixel. When creating a classified aerial photo, each vegetation class was treated individually at first, since different misclassification probabilities exist for each vegetation class. A complete classified aerial photo $\left(\hat{P}_{\text {cap }}\right)$ was finally obtained by merging each single classification. In the same way, classified satellite images $\left(\hat{P}_{\mathrm{csi}}\right)$ were generated by using their related confusion matrix. The perfect datasets with 13 vegetation classes were used for this purpose. With regard to the rest, the simulation was made in the same way as the classified aerial photo image.

The field surveys $\left(\hat{P}_{f \mathrm{~s}}\right)$ at time t0 and time $\mathrm{t} 1$ were generated by randomly selecting $5 \%$ of the existing lichen cells to represent the field measurements. The classifications of the lichen cover were here assumed to be correct at each sampling point being surveyed.
When generating artificial satellite images $\left(\hat{P}_{\text {sat }}\right)$, simulations from the perfect datasets $\left(U_{0}^{*}\right.$ and $\left.U_{1}^{*}\right)$ were made by using the mean vector and the covariance matrix for each vegetation type. However, within this study the generalized NDVI method did not seem to cause any significant change. For that reason known error propagation rules were used to verify if the NDVI method could function as a change detection method. By using the mean vectors and the covariance matrices for the used intensities, the standard deviations of the NDVI values, the NDVI differences, the standard errors of these differences, and the percentage share of cells having a significant change were calculated. This theoretical study confirmed that the NDVI method implemented in this study was inapplicable.

\section{Alarms}

In this study, 2 indicators of alarm were defined as follows: - If more than $15 \%$ of the total number of lichen cells have lost all their lichen content, an alarm is triggered.

- A pixel (cell) where the amount of lichen has decreased by more than $50 \%$ is considered to be affected. If the number of affected cells is more than $30 \%$ of the total number of lichen cells, an alarm is triggered.

In the methods using classified aerial photos or field surveys for change detection, the amount of lichen at each cell was estimated. This means that both the alarm indicators above could be used.

Since a classified satellite image only contains one lichen class, the change detection with this method could only be measured by the amount of disappearing lichen cells. This means that only the first of the 2 indicators could be used.

\section{Risk Assessment}

The suitability of the dataset $\hat{P}$ is here defined as

$\bar{S}_{\hat{p}}=\operatorname{Dist}_{2}\left(\hat{d}-d^{*}\right)=\operatorname{Dist}_{2}\left(f(\hat{P})-f\left(U^{*}\right)\right)$

In the sections above, we have described the procedures of estimating $f(\hat{P})$ (for the different monitoring systems and $f\left(U^{*}\right)$.

In this study, the suitability of the dataset is defined as its expected utility EU,

$$
E U=\sum_{i} \operatorname{Pr}\left(\xi_{i}\right) \cdot \mathrm{u}_{i}
$$

where $\operatorname{Pr}\left(\xi_{i}\right)$ is the probability of state $\xi_{i}$ occurring $u_{i}$ is the costs and benefits associated with state $\xi_{i}$

Assume that we have a decision-making process with a finite number, $n$, of decisions. We then have $n * n$ possible future states, namely the combinations of the actual decision being made and the correct decision based on correct information. We may then construct a probability matrix, PM, of the dimension $n^{*} n$, where

$$
\mathrm{PM}_{i, j}=\operatorname{Pr}\left(\hat{d}=d_{i} \wedge d^{*}=d_{j}\right)
$$

In our case, we have $\mathrm{d}_{1}=$ Alarm and $\mathrm{d}_{2}=$ No alarm. Our PM matrix is then

$$
P M=\left(\begin{array}{ll}
\operatorname{Pr}(\text { Correct alarm }) & \operatorname{Pr}(\text { False alarm }) \\
\operatorname{Pr}(\text { Missed alarm }) & \operatorname{Pr}(\text { Correct no action })
\end{array}\right)
$$


If, for each of these future states, we estimate the corresponding costs and benefits $\mathrm{u}_{\mathrm{i}, \mathrm{j}}$, we may then estimate our expected utility as

$$
E U=\sum_{i} \sum_{j} P M_{i, j} \cdot u_{i, j}
$$

The utility of each action (correct alarm, false alarm, etc) is estimated as a vector consisting of the environmental utility and monetary utility. A positive utility corresponds to an improvement in the environmental quality or a financial profit, while a negative utility corresponds to environmental or monetary costs. The main reason for expressing the utilities as a vector is the difficulty in transforming environmental improvements and costs into monetary terms.

For all monitoring methods, the environmental utility matrix is given by

$$
U M_{e n v}=\left(\begin{array}{cc}
\text { Gain due to restrictions } & \text { Costs due to minor land degradation } \\
\text { Costs due to major land degradation } & \text { Costs due to minor land degradation }
\end{array}\right)
$$

In this case study, it is assumed that when an alarm is triggered, a more detailed investigation is carried out in order to ensure that it is not a false alarm. For monitoring methods with good performance, this action may be omitted. If the detailed investigation confirms that land degradation is taking place, restrictions on the land use are to be implemented. For cost estimation, this implies a classic problem, namely whether or not costs for external parties should also be included.

For each monitoring method, the following monetary utility matrix is estimated:

$$
U M_{\text {mon }}=\left(\begin{array}{cc}
\mathrm{c}_{1}+\mathrm{c}_{2}+\mathrm{c}_{3} & \mathrm{c}_{1}+\mathrm{c}_{2} \\
\mathrm{c}_{1} & \mathrm{c}_{1}
\end{array}\right)
$$

where

$\mathrm{c}_{1}$ is the cost of monitoring the lichen cover

$c_{2}$ is the cost of a more detailed investigation, in the case of an alarm

$\mathrm{c}_{3}$ is the cost of implementing restrictions on land use

On the basis of a limited number of interviews, the environmental and monetary utilities described above have been estimated. The variations among the answers are in some cases considerable, indicating an uncertainty in the estimates that must be considered in evaluating the figures.

\section{RESULTS}

The results of the simulations are summarized by the following probability matrices, PM,

$$
\begin{aligned}
& P M_{\text {FieldSurveys }}=\left(\begin{array}{cc}
0.33 & 0 \\
0 & 0.67
\end{array}\right) \\
& P M_{\text {ClassifiedArealPhoto }}=\left(\begin{array}{cc}
0.33 & 0 \\
0 & 0.67
\end{array}\right) \\
& P M_{\text {ClassifiedSatelliteImages }}=\left(\begin{array}{ll}
0.27 & 0.64 \\
0.06 & 0.03
\end{array}\right)
\end{aligned}
$$

According to the simulations, the probability of a significant change in the land cover is $33 \%$. However the field surveys, as well as the photo interpretation, gave correct alarms for all the investigated situations. The use of classified satellite images did not perform equally well.

For the 3 different monitoring methods studied, the utilities shown in Table 1 were estimated. Note that the environmental utility is expressed along a relative scale, while the monetary costs are expressed in monetary units.

Table 1 shows that the field surveys and the interpretation of aerial photographs provide an equally good environmental gain. Moreover, considering the monetary costs, the interpretation of aerial photographs is, in this case, slightly less expensive than the field surveys. The classification of satellite images is here the least expensive method, but also provides the lowest environmental gain.

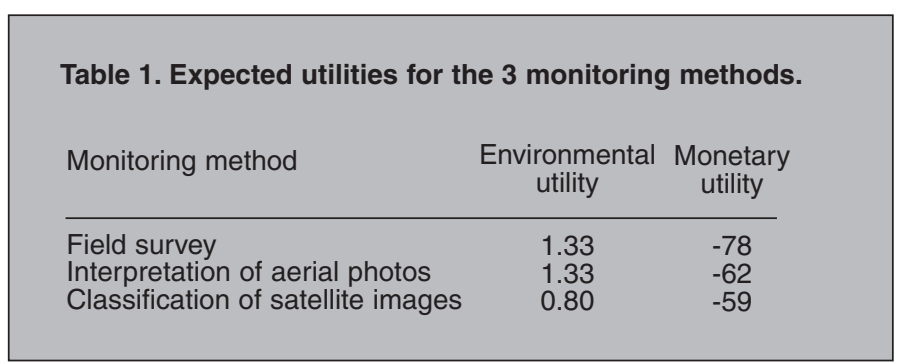

\section{DISCUSSION}

This paper describes a method for measuring fitness for use within decision making by quantifying usability in terms of risk and cost. DeBruin et al. (11), as well as Crosetto and Tarantola (10) and Aerts (12), introduce somewhat similar concepts, whereas other related studies mainly focus on describing how to analyze uncertainties associated with model outputs.

It is shown in this study that the expected utility consists of 2 parts, namely the probability of land degradation and the costs and benefits of triggering alarms. Both parts are sitespecific. This means that the presented results are sitespecific, and no conclusion can be drawn concerning what monitoring method is preferable in general. It should also be stressed that no attempts have been made to optimize any monitoring method, although such procedures may be designed within the framework described in this paper.

One should also be aware of the fact that the method based on post-classification is questioned by Singh (21). Singh's paper discusses and compares different digital change detection techniques, and concludes that the postclassification technique seems to have the lowest change classification accuracy. That result is verified in this study.

Change detection based on NDVI differences was found to be inapplicable in the case study. When trying to implement the NDVI method, it was found both empirically and theoretically, that this method was too weak. Most likely this is due to the use of a change detection strategy that was far too generalized. Another reason might be that more information than that provided by the mean vector and the covariance matrix is needed to generate adequate satellite images. Perhaps other additional external information is required as well. Moreover, other images with sharper differences between the lichen classes might be required. Furthermore, Nordberg 
and Allard (2) show that changes in the lichen cover can be detected in Landsat TM data using an NDVI differencing technique with an overall accuracy of $77 \%$, indicating that better results may be obtained by implementing their method in an improved way.

One conclusion that can be drawn is that standardized quality descriptors as such say nothing about the possibility of triggering alarms in monitoring situations. However, on the other hand, it is shown that such descriptors can be processed further and can be useful when evaluating monitoring systems.

Since confusion matrices for the classified satellite images, as well as for the classified aerial photos, were lacking, these were created on the basis of interviews and reviews of earlier studies. However the material that forms the basis for the creation of these confusion matrices is somewhat limited.

Quality specifications of source data form the basis for the production of the different datasets used in this study. For this reason the method depends on the availability of such information. The case study is a significant example where standardized quality measures are extremely useful, but sometimes not enough. This is especially obvious when studying the change detection method based on the NDVI differences. It is worth mentioning that even though the confusion matrix is a de facto standard quality descriptor, it is not always the case that it will be produced.

When generating an artificial perfect dataset $\left(U^{*}\right)$, spatial microsimulation based on cellular automata has been used. Microsimulation is very sensitive to variations in the models. This problem is usually handled by aligning the simulations to macroconstraints, such as the overall probability of land degradation. Such constraints have not been applied here, indicating that further refinement may be necessary.

The number of simulation runs required for obtaining robust results has not been investigated. In this study, 30 microsimulation runs were used. It is believed, however, that the uncertainty in the microsimulation model is greater than the uncertainty caused by the limited number of simulation runs.

The method can not only be used for comparing different strategies for environmental monitoring. Of equal interest might be the possibility of assessing the value of different datasets or optimizing a given monitoring method, for instance the number of sampling points in field surveys. The key issue for this kind of analysis is the identification of sources of uncertainty.

In this study, the expected utility is used as a measure of the usability of a dataset produced by a monitoring method. The advantage of this approach is that it clearly demonstrates the connection between data quality, decisions and environmental and financial costs and benefits. This is also a drawback, since some estimates of utilities are very uncertain and vary considerably among different persons. This demands a closer connection between engineering and environmental economics in future studies.

The relationships between costs and probabilities for different types of events can also be used for optimizing a monitoring system with respect to costs. If, for instance, the environmental cost of missing an alarm is very high, monitoring methods that do not miss such alarms are preferred, providing that the probability of land degradation is sufficiently high. If, in addition, the cost of implementing a restriction on land use is considered to be very dominant, the costs related to the monitoring methods as such are of less importance.
An important issue not being dealt with in this pilot study is the type of decision that should be considered for optimization. In many organizations, decisions are taken gradually in sequence and perhaps not always in a rational manner. This will of course influence the data usability function being used, and there might also be several occasions when the expected utility measure is not suitable.

\section{CONCLUSIONS}

The main aim of this paper was to study a method for evaluating the impact of data quality and model uncertainties within the decision phase, with special emphasis on the risks and costs associated with the design of different environmental monitoring systems. The following conclusions can be drawn from the study performed:

- Standardized quality measures are extremely useful for evaluating the usability of environmental monitoring methods. However, these measures cannot be used directly as indicators. Instead they have to be transformed into a domain understood by the user. The method proposed in this paper has a conceptually different approach compared to the approach suggested by ISO for evaluating the suitability of a certain dataset.

- As a suitability measure for environmental monitoring methods, the expected environmental gains and monetary costs have been used. When estimating such gains and costs, special attention must be paid to the following:

- If synthetic data are constructed using spatial microsimulation techniques, macroconstraints such as a priori probabilities should be used for aligning the simulation model.

- If synthetic data are constructed using Monte-Carlo simulation techniques, good models of the errors and uncertainties must be used. The standardized quality measures proposed by ISO have not proved to be insufficient.

- The utility function associated with the outcome of the decisions must be studied in more detail before the approach presented here can be used in real situations. It is especially important to decide which external monetary costs should be included in the calculations, for instance, costs due to restrictions on land use. It is of equal importance to have a concrete model for the environmental gains and costs associated with different scenarios.

\section{References and Notes}

1. Swedish EPA 1999. Miliömålen i Sverige: System med indikatorer för nationell uppföljning av miljökvalitetsmålen. (Environmental Quality Objectives in Sweden: Indicator System for National Monitoring of the Environmental Quality Objectives) Report 5006. Swedish Environmental Protection Agency, Stockholm, Sweden. (In Swedish with English summary.)

2. Nordberg, M.-L. and Allard, A. 2002. A remote sensing methodology for monitoring lichen cover. Can. J. Remote Sens. 28, 262-274.

3. Müller-Wille, L. 1975. Changes in lappish reindeer herding in Northern Finland caused by mechanisation and motorization, In: Proc. First International Reindeer and Caribou Symposium. Luick, JR et al. (eds). Biological papers of the University of Alaska, Special Report No 1, 122-126.

4. Cornélis, B and Brunet, S. 2002 A policy-maker point of view on uncertainties in spatial decisions, In: Spatial Data Quality. Shi, W., Fisher, P.F. and Goodchild, M.F. (eds). Taylor \& Francis, pp. 168-185.

5. Mowrer, H.T. and Congalton, R.G. (eds). 2000. Ouantifying Spatial Uncertainty in Natural Resources: Theory and Applications for GIS and Remote Sensing. Ann Arbor Press. 
6. Story, M. and Congalton, R. 1986. Accuracy assessment: A user's perspective.

7. Lillesand, T.M. and Kiefer, R.W. 1994. Remote Sensing and Image Interpretation, 3rd ed. John Wiley \& Sons.

. Goodchild, M.F. 2000. Communicating the results of accuracy assessment: metadata, digital libraries, and assessing fitness for use. In: Quantifying Spatial Uncertainty in Natural Resources: Theory and Applications for GIS and Remote Sensing, Mowrer, H.T. and Congalton, R.G. (eds). Ann Arbor Press, pp. 3-15.

9. ISO 2002. Geographic Information: Quality Principles. International Standard 19113. 0. Crosetto, M. and Tarantola, S. 2001. Uncertainty and sensitivity analysis: Tools for GIS-based model implementation. Int. J. Geogr. Info Sci. 15, 415-437.

11. DeBruin, S., Bregt, A. and Van De Ven, M. 2001. Assessing fitness for use: the expected value of spatial data sets. Int. J. Geogr. Info. Sci. 15, 457-471.

12. Aerts, J.C.J.H. 2002. Spatial Decision Support for Resource Allocation: Integration of Optimization, Uncertainty Analysis and Visualization Technique. PhD Thesis, University of Amsterdam, The Netherlands.

13. Frank, A. and Grünbacher, A. 2002. What is relevant in a dataset? In: Proc. $5^{\text {th }}$ AGILE Conference on Geographic Information Science, pp. 259- 263

14. Krek, A. 2002. Cognition, value and price of geographic information. In: Proc. $5^{\text {th }}$ AGILE Conference on Geographic Information Science, pp. 271- 277.

15. Renman, G. 1989. Barmarkskörning på fjällen. Effekter av körning med terränghjulingar på mark och vegetation. (Driving on Bare Ground in the Mountanious Area. Effects of Cross-country Driving with Off-highway Vehicles) Swedish Environmental Protection Agency, Report 3598

16. Allard, A. 2001. Vegetation Changes in Mountainous Area: A Detailed Study by Aerial Photo-based Remote Sensing and Spectral Radiometry. Licensiate Thesis, Departmen of Physical Geography and Quaternary Geology, Stockholm University, Sweden.

17. Pegau, R.E. 1970. Effect of reindeer trampling and grazing on lichens. J. Range Mgm 23, 95-97.

18. Leader-Williams, N., Smith, R.I.L. and Rothery, P. 1987. Influence of introduced reindee on the vegetation of South Georgia: Results from a long-term exclusion experiment. $J$ Appl. Ecol. 24, 801-822

9. Tömmervik, H. and Lauknes, I. 1987. Mapping of the reindeer ranges in the Kautokeino area, Northern Norway, using landsat 5/TM data. Rangifer 7, 2-14

20. Allard, A., Ihse, M. and Nordberg M.-L. 1998. Vegetationsförändringar i fjällen: metodstudier i norra fjällen med hjälp av IRF-flygbilder och satellitbilder. (Vegetation Changes in the Mountanious Area: Studies of methods in the Northern Area Usin Near-infrared Sensitive Areal Color Film and Satellite Imagery) Research Report 109 $64 \mathrm{p}$. Department of Physical Geography, Stockholm University. Also published as World Wide Fund for Nature report, WWF Report 5:98, 64 pp. (In Swedish with English abstract.)

21. Singh, A. 1989. Digital change detection techniques using remotely-sensed data. Int. J. Remote Sens. 10, 989-1003.

22. Eriksson, O., Niva, M. and Raunistola, T. 1998. Långtidsförändringar av fjällväxtlighet. Några exempel från norra Lappland, (Long-time changes of mountain vegetation. Some examples from northern Lapplandia) In: Rapport från 1997 Ars Fjällforskningskonferens, Forskningsrådsnämnden (FRN), report 98, pp 184-198. (In Swedish).

23. Clarke, G.P. 1996. Microsimulation: an introduction. In: Microsimulation for Urban and Regional Policy Analysis. Clarke, G.P. (ed.). Pion. pp. 1-9.

24. Esko, J. 2002. Synthetic Microdata. Licentiate thesis, Luleå University of Technology, Sweden
Britt-Inger Rönnbäck, is a PhD student at the Division of Geographical Information Technology, Luleå University of Technology. She has an MSc in surveying, and a licentiate degree in geographic information technology. Before joining Luleå University of Technology she worked as a software developer and system analyst at the Swedish Space Corporation and at the Swedish Land Survey. She is planning to defend her PhD thesis autumn 2003. The thesis concerns spatial data quality and data usability. Her address: Department of Environmental Engineering, Geographical Information Technology, Luleå University of Technology, SE-971 87, Luleå, Sweden.

britt-inger.ronnback@sb.luth.se

Maj-Liz Nordberg, PhD, is a senior lecturer at the Department of Physical Geography and Quaternary Geology, Stockholm University carrying out research in applied remote sensing. Her current research focuses on various change detection aspects for monitoring purposes. The primary field site is the Swedish mountain range. Her address: Department of Physical Geography and Quaternary Geology, Stockholm University, SE-106 91, Stockholm, Sweden.

maj-liz.nordberg@natgeo.su.se

Anders Olsson, is a PhD student at the Division of Geographical Information Technology, Luleå University of Technology. His research deals with questions concerning spatial data quality and automatic methods for such assessments. His address: Department of Environmental Engineering, Geographical Information Technology, Luleå University of Technology, SE-971 87, Luleà, Sweden. anders.olsson@sb.luth.se

Anders Östman, is a professor at the Division of Geographic Information Technology, Luleå University of Technology, and the head of the division. He has an MSc in surveying and a PhD in photogrammetry. He has also worked for Intergraph, an American GIS software vendor. Since 1993, Anders Östman has gradually established the research and educational facilities in GIS at Luleå University of Technology, where he received a full professorship in 1997 His research concerns spatial data quality. His address: Department of Environmental Engineering, Geographical Information Technology, Luleå University of Technology, SE-971 87, Luleå, Sweden. anders.ostman@sb.luth.se 